\title{
SUPERVISED CLASSIFICATION OF MULTIPLE VIEW IMAGES IN OBJECT SPACE FOR SEISMIC DAMAGE ASSESSMENT
}

\author{
M. Gerke \\ University of Twente, Faculty of Geo-Information Science and Earth Observation - \\ ITC, Department of Earth Observation Science, Hengelosestraat 99, P.O. Box 217, 7500AE Enschede, Netherlands
}

Working Groups I/2, III/1, III/4, III/5

KEYWORDS: AdaBoost, classification, feature, fusion, learning, performance, point cloud, random trees

\begin{abstract}
:
Classification of remote sensing image and range data is normally done in 2D space, because anyhow most sensors capture the surface of the earth from a close-to vertical direction and thus vertical structures, e.g. at building facades are not visible anyways. However, when the objects of interest are photographed from off-nadir directions, like in oblique airborne images, the question on how to efficiently classify those scenes arises. In this paper a study on classification in 3D object space is presented: image features from individual oblique airborne images, and 3D geometric features derived from matching in those images are projected onto voxels. Those are segmented and classified. The study area is Port-Au-Prince (Haiti), where images have been acquired after the earthquakes in January 2010. Results show that through the combination of image evidence as realized by the projection into object space the classification becomes more accurate compared to single image classification.
\end{abstract}

This contribution was selected in a double blind review process to be published within the Lecture Notes in Computer Science series (Springer-Verlag, Heidelberg).

Photogrammetric Image Analysis

Volume Editors: Stilla U, Rottensteiner F, Mayer H, Jutzi B, Butenuth M

LNCS Volume: 6952

Series Editors: Hutchison D, Kanade T, Kittler J, Kleinberg JM, Kobsa A, Mattern F, Mitchell JC, Naor M,

ISSN: $\quad$ Nierstrasz $\quad$ 0302-9743

The article is accessible online through www.springerlink.com. 